\title{
Impact of a time-dependent background error covariance matrix on air quality analysis
}

\author{
E. Jaumouillé ${ }^{1}$, S. Massart ${ }^{1}$, A. Piacentini ${ }^{1}$, D. Cariolle ${ }^{1}$, and V.-H. Peuch ${ }^{2}$ \\ ${ }^{1}$ CERFACS/CNRS-URA 1875, 31057 Toulouse, France \\ ${ }^{2}$ CNRM-Game/CNRS-URA 1357, 31057 Toulouse, France \\ Correspondence to: S. Massart (sebastien.massart@cerfacs.fr)
}

Received: 24 February 2012 - Published in Geosci. Model Dev. Discuss.: 23 April 2012

Revised: 3 August 2012 - Accepted: 6 August 2012 - Published: 6 September 2012

\begin{abstract}
In this article we study the influence of different characteristics of our assimilation system on surface ozone analyses over Europe. Emphasis is placed on the evaluation of the background error covariance matrix (BECM). Data assimilation systems require a BECM in order to obtain an optimal representation of the physical state. A posteriori diagnostics are an efficient way to check the consistency of the used BECM. In this study we derived a diagnostic to estimate the BECM. On the other hand, an increasingly used approach to obtain such a covariance matrix is to estimate it from an ensemble of perturbed assimilation experiments. We applied this method, combined with variational assimilation, while analysing the surface ozone distribution over Europe. We first show that the resulting covariance matrix is strongly time (hourly and seasonally) and space dependent. We then built several configurations of the background error covariance matrix with none, one or two of its components derived from the ensemble estimation. We used each of these configurations to produce surface ozone analyses. All the analyses are compared between themselves and compared to assimilated data or data from independent validation stations. The configurations are very well correlated with the validation stations, but with varying regional and seasonal characteristics. The largest correlation is obtained with the experiments using time- and space-dependent correlation of the background errors. Results show that our assimilation process is efficient in bringing the model assimilations closer to the observations than the direct simulation, but we cannot conclude which BECM configuration is the best. The impact of the background error covariances configuration on four-days forecasts is also studied. Although mostly positive, the impact depends on the season and lasts longer during the winter season.
\end{abstract}

\section{Introduction}

Data assimilation techniques are frequently used in many fields of application especially in Geosciences, such as weather forecasting and oceanography (Swinbank et al., 2003). They are used to provide an estimate of the physical state of a system consistent with the input information. This information comes from an a priori estimate of the physical state (also called the background) and from observations linked to the current state of the system. The assimilation output is the best estimate of the physical state given the input information. It is known as the analysis. The process that creates an analysis also involves the observational and background error covariance matrices. These matrices determine the respective weights in the analysis given to each of these two pieces of information. The background matrix determines the spatial distribution of the correction introduced in the analysis and the balance between the control variables of the system. An optimal state can only be obtained by the assimilation process if these matrices are correctly specified (Reichle, 2008). But direct determination of the background error covariance matrix (BECM) requires information that is not directly available. It is thus important to find the appropriate way to estimate the BECM, as it is known to strongly influence the assimilation results.

Recent studies of Massart et al. (2012) or Elbern et al. (2010) investigate the evaluation of the BECM but for global atmospheric chemistry. In air quality applications, several methodologies are currently used, but BECMs are mainly obtained with ensemble Kalman filter (EnKF) approaches. For example, Coman et al. (2012) used an EnKF in their analysis of partial lower tropospheric ozone columns to provide estimations of the background errors. 
Constantinescu et al. (2007) have also investigated the EnKF for the simulation of air pollution in the Northeastern United States. Alternatively, in atmospheric chemistry as in other geophysical applications, one can use a statistical interpolation to specify an anisotropic and heterogeneous BECM (Blond et al., 2003) or can combine an ensemble approach with a variational data assimilation approach (Massart et al., 2012; Desroziers et al., 2008; Buehner, 2005). We have used this approach for global ozone analyses, and we examine it here to provide a time-dependent BECM for a regional chemical application.

The goal of this paper is to investigate the impact of an ensemble-derived time-dependent BECM in regional atmospheric chemical data assimilation. This study contributes to the European MACC project (monitoring atmospheric composition and climate) whose one of its goals is to provide information services on European air quality. Different chemistry and transport models (CTMs) contribute to this project to produce daily air quality forecasts for Europe and reanalyses of European air quality. In this study we use Mocage (MOdèle de Chimie Atmosphérique à Grande Echelle), the nested-domains CTM developed at Météo-France (Peuch, 1999). Mocage is combined with an adapted version of the Centre Européen de Recherche et Formation Avancée en Calcul Scientifique (Cerfacs) Valentina variational data assimilation system (Massart et al., 2005) to produce daily analyses of surface ozone over Europe. The Mocage-Valentina variational data assimilation system is combined with an ensemble method. We first estimate the time-dependent BECMs and in particular their variance and their correlation elements. We build several configurations of the BECM, some of them derived from the ensemble estimate. Analyses resulting from the different experiments using the different BECM models are then studied and compared. The impact of the chosen model on four day forecasts is also investigated.

The structure of this paper is as follows. In Sect. 2, the Mocage-Valentina chemical assimilation system is presented, and results on an air quality case study are developed in Sects. 3 and 4. In Sect. 5, several selected experiments each using a specific background error covariance matrix are described and the impact of these experiments on the analysis and forecasts is studied. Conclusions and a discussion are presented in Sect. 6.

\section{The background error covariance matrix in the assimilation process}

In this section, the assimilation process is briefly presented. The main properties of the BECM are also given and the chosen methods to estimate the BECM are described.

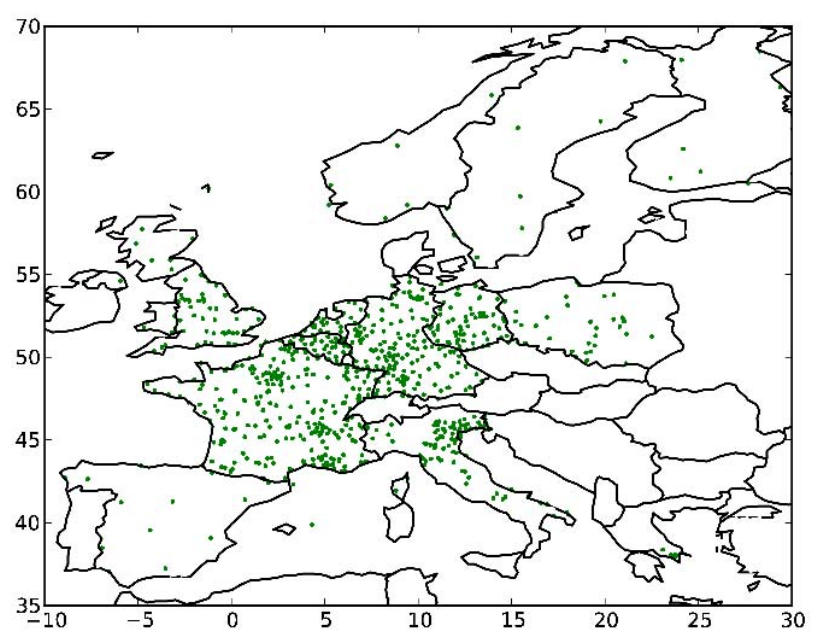

Fig. 1. Example of the location of MACC ozone ground-based stations for the 1 July 2010 .

\subsection{Assimilation process}

The purpose of any data assimilation method is to compute the best estimate of the unknown true state of the system studied. The estimate is usually based on the optimal combination of observations and of other sources of information all gathered in a priori information. When the a priori information comes from a model, it is expressed on the model grid as the background vector $\mathbf{x}^{\mathbf{b}}$ associated with the B BECM. All the information from observations is gathered in the $\mathbf{y}^{\mathbf{0}}$ vector, associated with the $\mathbf{R}$ observation error covariance matrix (OECM). In order to compare any state $\mathbf{x}$ to the observations, the possibly non-linear $H$ observation operator is generally introduced.

The variational approach for resolving the assimilation problem consists in minimizing the variational cost function

$$
\begin{aligned}
J(\mathbf{x}) & =\frac{1}{2}\left(\mathbf{x}-\mathbf{x}^{\mathbf{b}}\right)^{T} \mathbf{B}^{-1}\left(\mathbf{x}-\mathbf{x}^{\mathbf{b}}\right) \\
& +\frac{1}{2}\left(\mathbf{y}^{\mathbf{o}}-H \mathbf{x}\right)^{T} \mathbf{R}^{-1}\left(\mathbf{y}^{\mathbf{o}}-H \mathbf{x}\right),
\end{aligned}
$$

that both quantifies the difference between the current state $\mathbf{x}$ and the background, and the difference between the observations and the equivalent in the observations space of the model state $\mathbf{x}$ (Talagrand, 1997). Each difference is normalized by its own error specified respectively in the BECM and the OECM.

The optimal state $\mathbf{x}^{\mathbf{a}}$ is the one which minimizes the cost function of Eq. (1). This particular model state is called the analysis. The analysis will be strongly affected by the error covariance matrices. As an example, assuming high values of the background error will reduce the weight of the background term in the cost function of Eq. (1) and will make the analysis closer to the observations (in the observations space). 


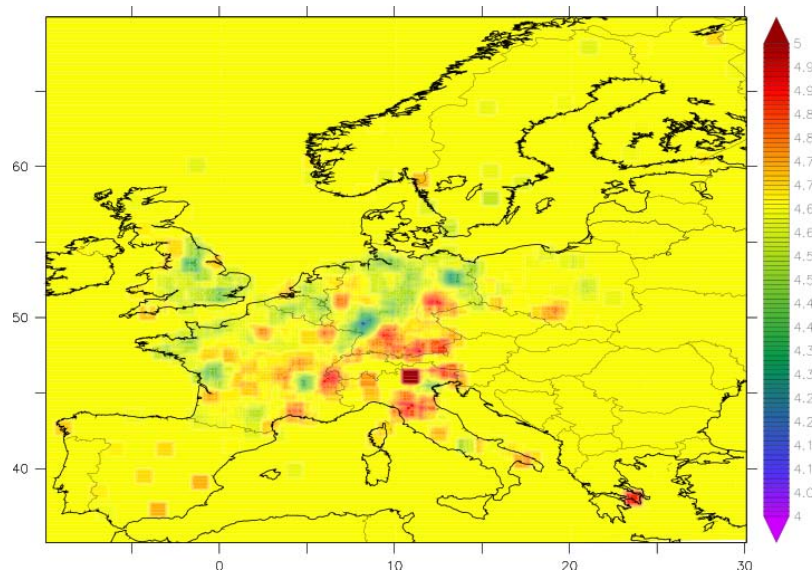

(a)

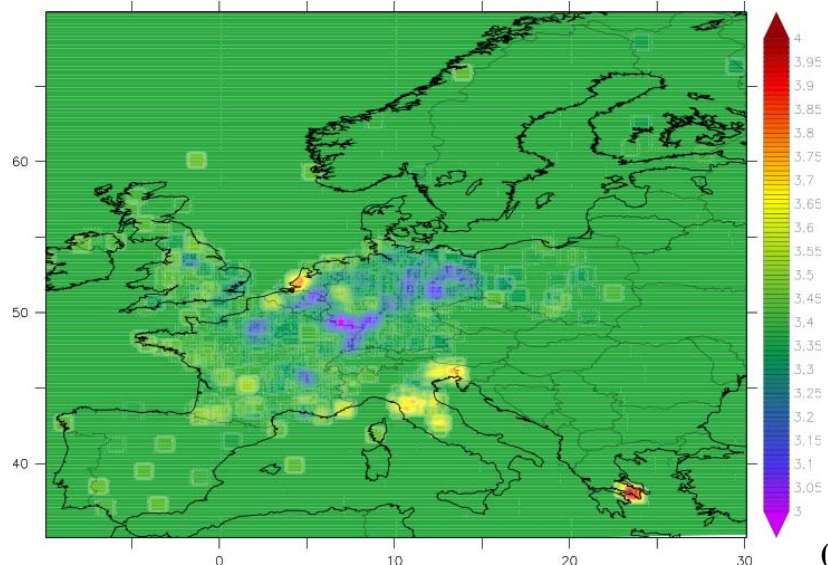

(b)

Fig. 2. Diagnosed standard deviation from a posteriori diagnostics (ppb), averaged in the period between 1 and 10 July 2010 (a) and the period between the 1 and 10 December 2010 (b).

In the case of a linear observation operator, $H$ can be associated with its matrix form $\mathbf{H}$ and the analysis can be expressed as

$\mathbf{x}^{\mathbf{a}}=\mathbf{x}^{\mathbf{b}}+\mathbf{B} \mathbf{H}^{T}\left(\mathbf{R}+\mathbf{H B} \mathbf{H}^{T}\right)^{-1}\left(\mathbf{y}^{\mathbf{o}}-H \mathbf{x}^{\mathbf{b}}\right)$.

Equation (2) shows that the analysis is equal to the background plus a correction proportional to the innovation vector $\mathbf{d}_{\mathbf{b}}^{\mathbf{0}}=\mathbf{y}^{\mathbf{0}}-H \mathbf{x}^{\mathbf{b}}$. The correction is then normalized by $\left(\mathbf{R}+\mathbf{H B H}^{T}\right)^{-1}$. The correction is reintroduced to the model space through $\mathbf{H}^{T}$ and finally multiplied by $\mathbf{B}$. Here, the BECM matrix, through its covariances, spreads over the model space the information coming from the observations space and extrapolated by $\mathbf{H}^{T}$ to the model space. It includes a spatial dispersion around the measurement location and a spread between the control model variables. This behaviour reveals it is important to have an appropriate BECM.

The OECM contains errors in the observations process, such as instrumental errors and representativeness errors. A diagonal or block diagonal OECM is used in most data assimilation applications, which assumes that the observations are loosely correlated.

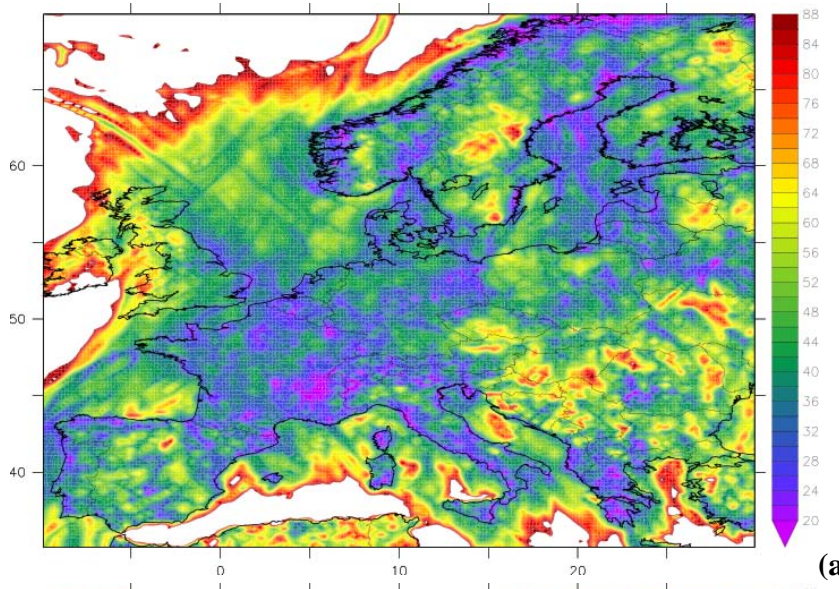

(a)

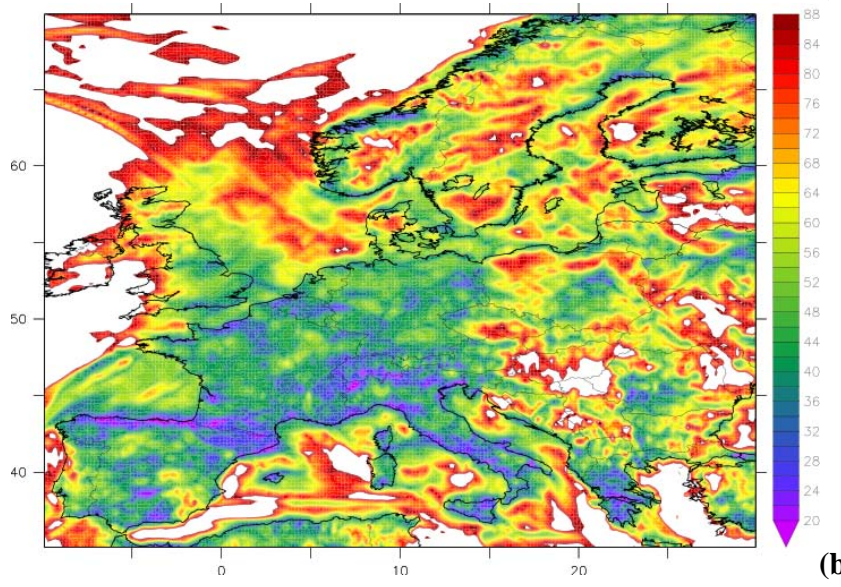

(b)

Fig. 3. Diagnosed length-scales (in $\mathrm{km}$ ) of the horizontal correlation of the forecast error, averaged in the period between 1 and 10 July 2010: west-east (a) and north-south (b) length-scales.

\subsection{The background error covariance matrix formulation}

The BECM is a key component in the data assimilation process. It contains two significant ingredients of our problem: how the observed information is spatially filtered and propagated, and how the control variables are correlated. The BECM is written as

$\mathbf{B}=\mathbb{E}\left(\boldsymbol{\varepsilon}_{\boldsymbol{b}} \boldsymbol{\varepsilon}_{\boldsymbol{b}}^{T}\right)$

that merges all the information on the background error $\varepsilon_{b}=\mathbf{x}^{\mathbf{b}}-\mathbf{x}^{\mathbf{t}}$ (assuming Gaussian statistics), where $\mathbf{x}^{\mathbf{t}}$ is the true state. Equation (3) assumes that the mathematical expectation $\mathbb{E}$ of $\boldsymbol{\varepsilon}_{\boldsymbol{b}}$ is equal to zero. This means an unbiased background error.

To directly calculate the BECM from Eq. (3), one would require the knowledge of the background vector $\mathbf{x}^{\mathbf{b}}$ and the true state vector $\mathbf{x}^{\mathbf{t}}$. But $\mathbf{x}^{\mathrm{t}}$ being unknown makes the direct computation of the BECM not possible. It must be estimated. Having an efficient and robust method for its estimation is a key ingredient of our assimilation system. The 

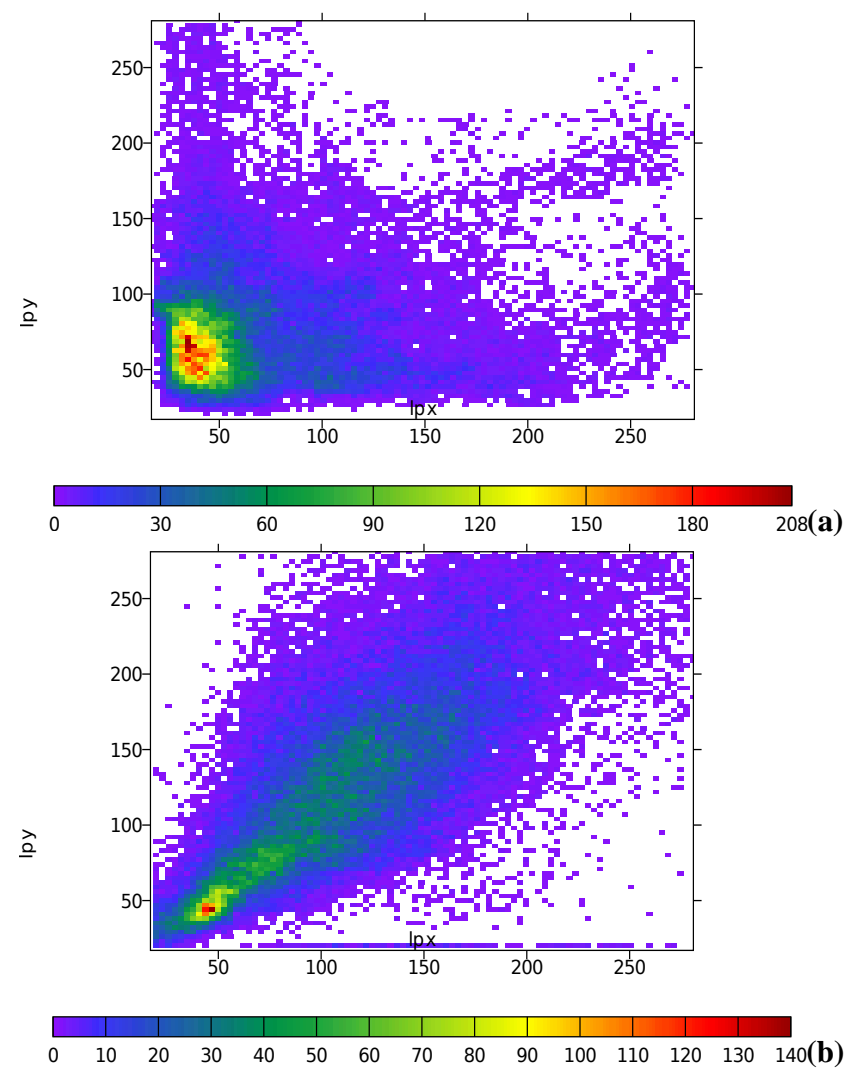

Fig. 4. Scatter diagram of length-scales (in $\mathrm{km}$ ) of the north-south and east-west correlations of the forecast error, averaged in the period between 1 and 10 July 2010 (a) and 1 and 10 December 2010 (b); lpx represents the west-east length-scales and lpy represents the north-south length-scales.

ensemble-based method combined with variational assimilation as described in the following section is one of those methods.

Due to its typical size in geoscience applications ( $\mathbf{B}$ is an $N \times N$ matrix where $N$ is the dimension of the model state vector, which can reach values greater than $10^{8}$ ), the BECM is usually written as

$\mathbf{B}=\Sigma \mathbf{C} \Sigma^{T}$

where $\Sigma$ corresponds to the diagonal matrix of standard deviations of the assimilated chemical species in each grid point of the model (equal to the square root of the variances) and C corresponds to the positive definite symmetric matrix of correlations.

In order to characterize the shape of the correlation functions, the length-scales diagnosis is often introduced (Daley, 1991; Pannekoucke et al., 2008; Belo Pereira and Berre, 2006). The length-scale is considered as a relevant indicator of the spatial dispersion around the observation location. The larger the length-scale, the more information given by the observation is dispersed. Most of the proposed formulations introduced to represent the length-scales suppose that
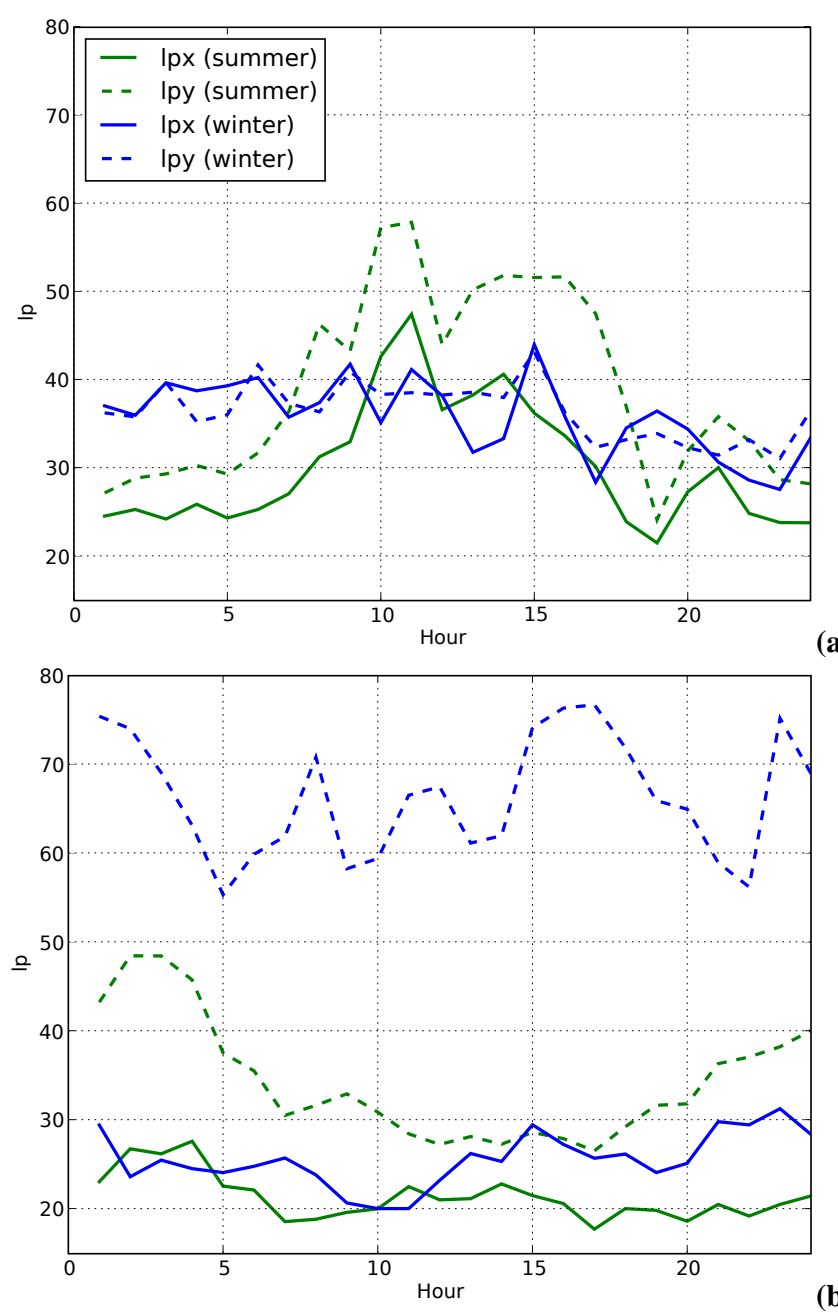

(b)

Fig. 5. Time series of estimated length-scales (in $\mathrm{km}$ ) of the correlation of the forecast error, hourly-averaged in the period between 1 and 10 July 2010 and in the period between 1 and 10 December 2010 in the grid cells of Paris (a) and Grenoble (b); lpx represents the west-east length-scales and lpy represents the north-south length-scales.

the correlation at the origin is a Gaussian function (Constantinescu et al., 2007; Pannekoucke and Massart, 2008; Weaver and Ricci, 2003). Thus the correlation $\rho$ between two points separated by a local distance of $\delta$ is

$\rho(\delta)=\exp \left(-\frac{\delta^{2}}{2 L^{2}}\right)$

where $L$ is the length-scale.

Estimating the BECM can then be reduced to the estimation of the diagonal of $\Sigma$ and the values of the length-scale required to compute $\mathbf{C}$. 


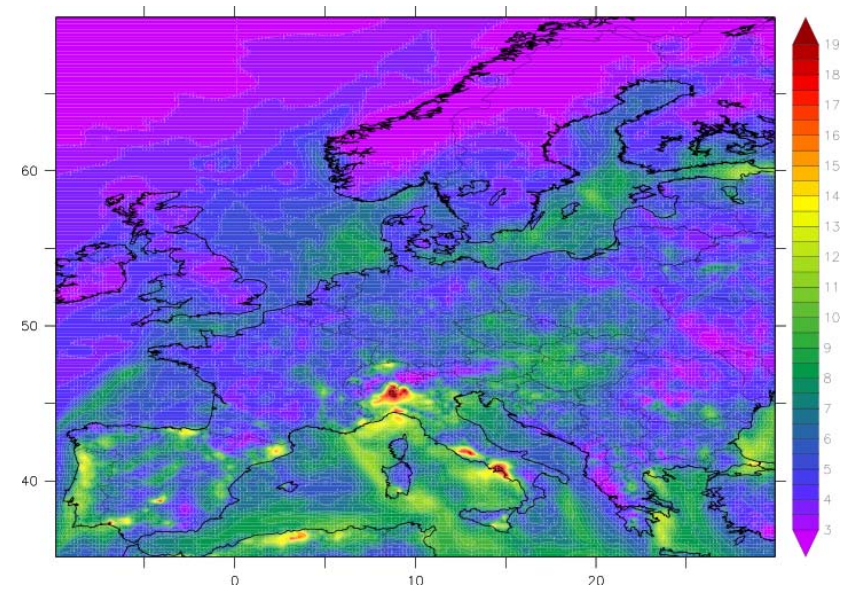

Fig. 6. Estimated standard deviations (in ppb), averaged in the period between 1 and 10 July 2010.
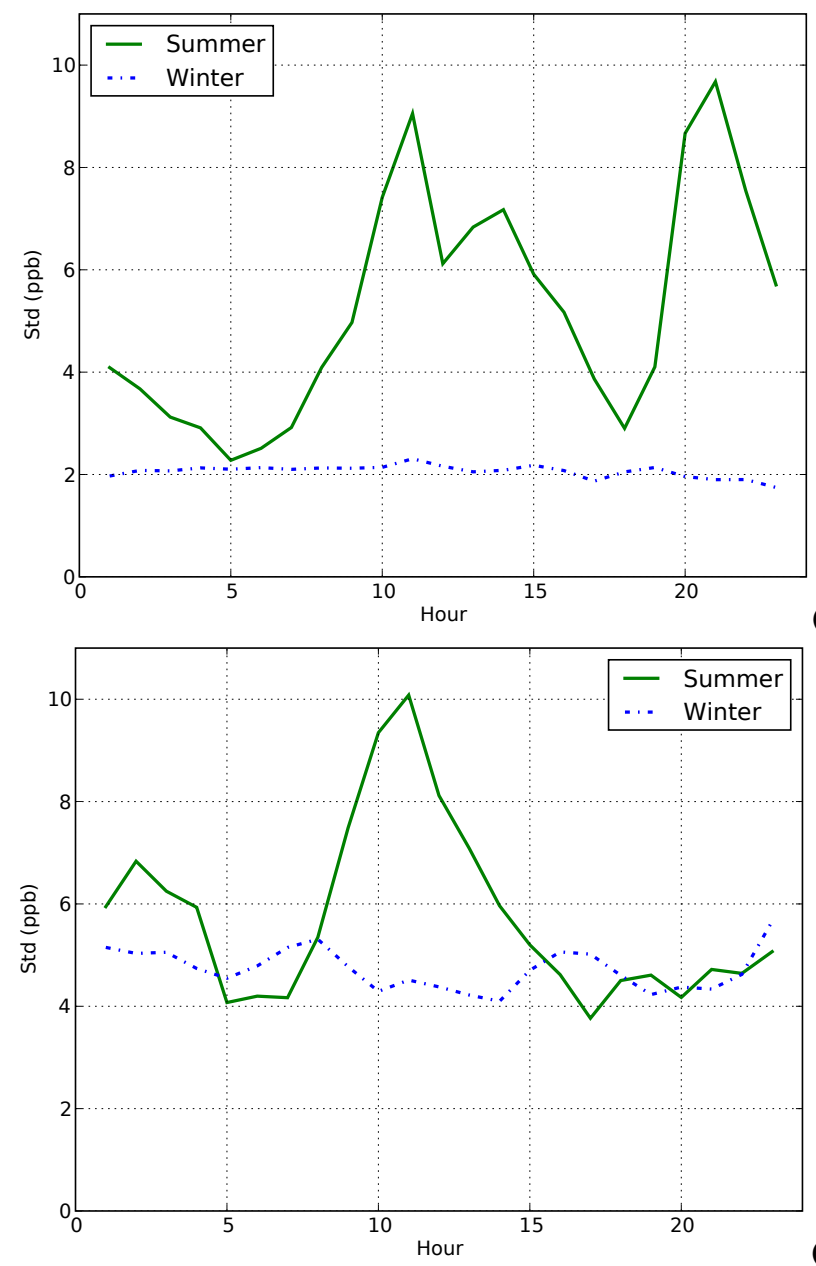

(a)

(b)

Fig. 7. Time series of estimated standard deviations (in ppb), hourly-averaged in the period between 1 and 10 July 2010 and in the period between 1 and 10 December 2010 in the grid cells of Paris (a) and Grenoble (b).
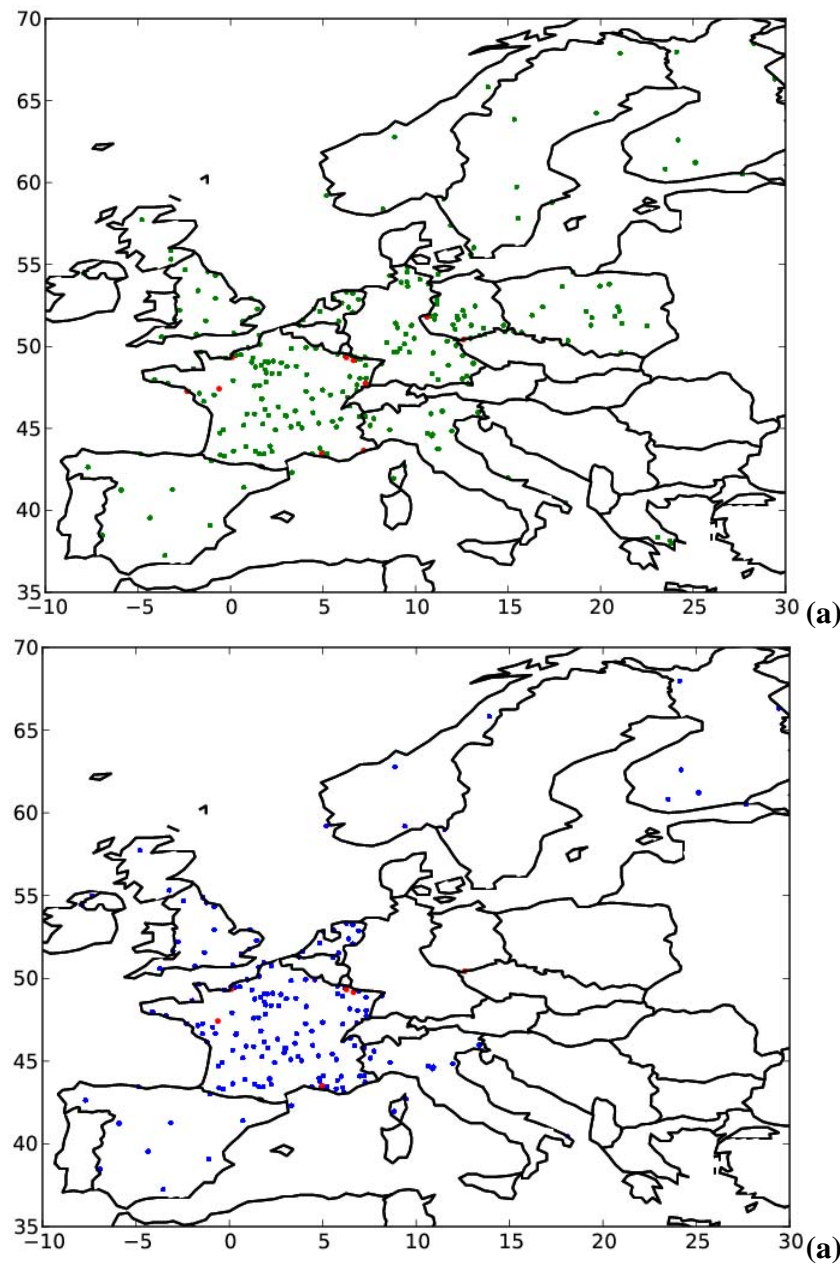

Fig. 8. Example of the location of MACC ozone rural ground-based stations, for the 1 July 2010 (a) and for the 1 December 2010 (b); the red points represent the validation stations.

\subsection{BECM derived from an ensemble approach}

One technique to estimate the BECM is to use an ensemble of variational assimilation experiments (Belo Pereira and Berre, 2006). This method consists in integrating the variational data assimilation system several times on the same period with perturbed parameters (e.g. initial conditions, forcing, etc.). Therefore, an ensemble of $n$ analyses $\left\{\mathrm{x}_{i}^{a}\right\}_{i=1, n}$ is obtained, where $n$ is the number of members of the ensemble. With a time-dependent model, forecasts are made from these analyses. These forecasts will form the background state for the next assimilation cycle. Then at each analysis time, an ensemble of backgrounds $\left\{\mathrm{x}_{i}^{b}\right\}_{i=1, n}$ is given. For each background state, its difference with the mean of the ensemble of backgrounds $\overline{\mathrm{x}^{b}}=\frac{1}{n} \sum_{i=1}^{n} \mathrm{x}_{i}^{b}$ gives an evaluation of the background error.

Considering the background error expression from Eq. (3), the BECM can be estimated from the ensemble of 
backgrounds by

$\mathbf{B} \simeq \frac{1}{n} \sum_{i=1}^{n}\left(\mathrm{x}_{i}^{b}-\overline{\mathrm{x}^{b}}\right)\left(\mathrm{x}_{i}^{b}-\overline{\mathrm{x}^{b}}\right)^{T}$.

The standard deviations and correlations matrices can be deduced from Eq. (6). The diagonal of the standard deviations matrix is estimated as

$\operatorname{diag}(\Sigma)=\sqrt{\frac{1}{n} \sum_{i=1}^{n}\left(\mathrm{x}_{i}^{b}-\overline{\mathrm{x}^{b}}\right)^{2}}$.

The correlation between a grid-point $k$ with another gridpoint $l$ can be written as

$\rho_{k l}=\frac{1}{n \Sigma_{k} \Sigma_{l}} \times \sum_{i=1}^{n}\left(\mathrm{x}_{i}^{b}(k)-\overline{\mathrm{x}^{b}(k)}\right)\left(\mathrm{x}_{i}^{b}(l)-\overline{\mathrm{x}^{b}(l)}\right)$

where $\Sigma_{k}$ (resp. $\Sigma_{l}$ ) is the background error standard deviations at the grid-point $k$ (resp. $l$ ). Assuming that $k$ and $l$ are two neighbour grid-points separated by a local distance equal to $\delta_{k l}$, the correlation obtained from Eq. (8) can be used to calculate the Gaussian length-scale using Eq. (5). Thus, the Gaussian length-scale is expressed as

$L_{k l}=\frac{\left|\delta_{k l}\right|}{\sqrt{-2 \ln \left(\rho_{k l}\left(\delta_{k l}\right)\right)}}$.

Belo Pereira and Berre (2006) have also proposed a relatively low computational cost formula for length-scale which can be used with a non Gaussian correlation:

$L=\sqrt{\frac{\left[\sigma\left\{\varepsilon_{b}(z)\right\}\right]^{2}}{\left[\sigma\left\{\partial_{z} \varepsilon_{b}(z)\right\}\right]^{2}-\left[\partial_{z} \sigma\left\{\varepsilon_{b}(z)\right\}\right]^{2}}}$

where $\sigma\left\{\varepsilon_{b}(z)\right\}$ is the standard deviation of $\varepsilon_{b}(z)$ and $\partial_{z}=$ $\partial / \partial_{z}$, the derivative along the latitude or the longitude. Note that the formula (10) requires the computation of the forecast-error standard deviations, its gradient and the standard deviation of the gradient of forecast error to calculate the length-scale.

A good estimate of the quantities in Eqs. (7) and (8) requires a large number of members to form the ensemble. But increasing the number of members increases the numerical cost. For high resolution state-of-the-art assimilation systems, the computational cost of a large number of members is too expensive. Constantinescu et al. (2007) have shown that the appropriate number of members varies, depending on the application, from about ten to some hundreds. Thus, to artificially increase the number of elements on which the statistics are computed, we can compute them on several assimilation cycles $c_{j}$. As an example, in the case of $m$ cycles, the diagonal of the standard deviations matrix can be rewritten as

$\operatorname{diag}(\Sigma)=\sqrt{\frac{1}{n \times m} \sum_{j=1}^{m} \sum_{i=1}^{n}\left(\mathrm{x}_{i}^{b}\left(c_{j}\right)-\overline{\mathrm{x}^{b}\left(c_{j}\right)}\right)^{2}}$.

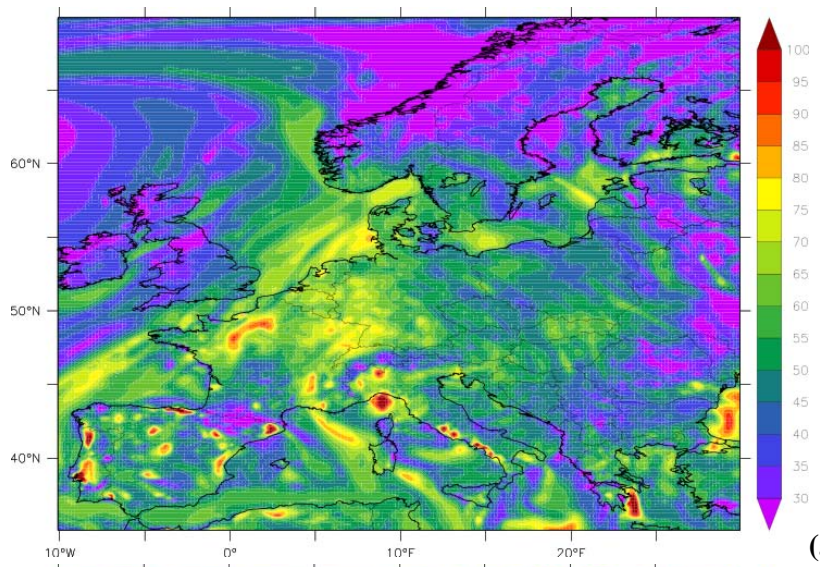

(a)

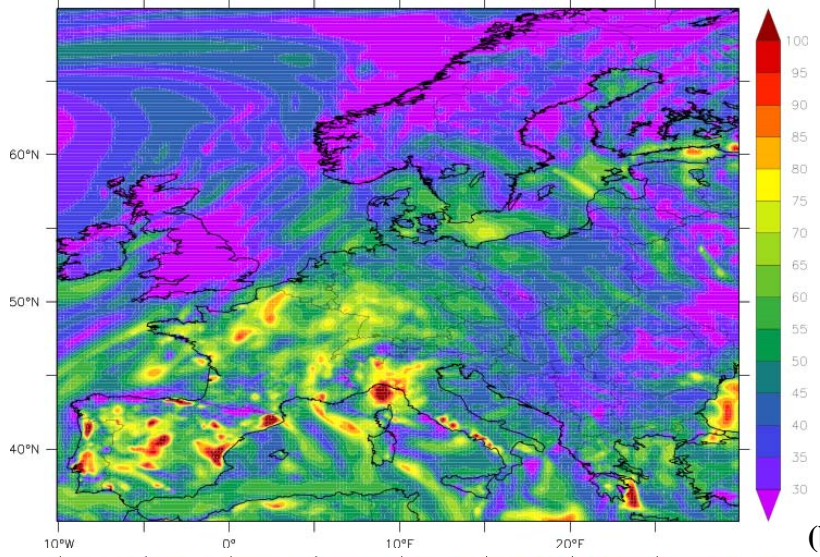

(b)

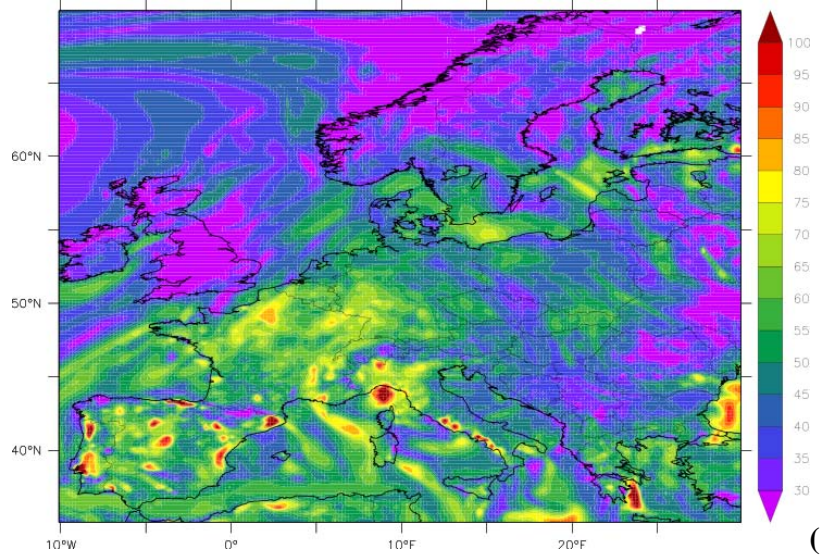

(c)

Fig. 9. Surface ozone concentration (in ppb) on the 7 July 2010 at 14:00:00 UTC for the (a) Direct simulation, (b) PERCEnT simulation, (c) OPER simulation, see Table 1 for details on the experiments.

Equation (8) can also be modified to take into account several cycles in the calculation of the length-scale. However, if a large number of cycles are used, the BECM estimate will lose its flow-dependent property. Depending on the system used, a compromise has to be found. 

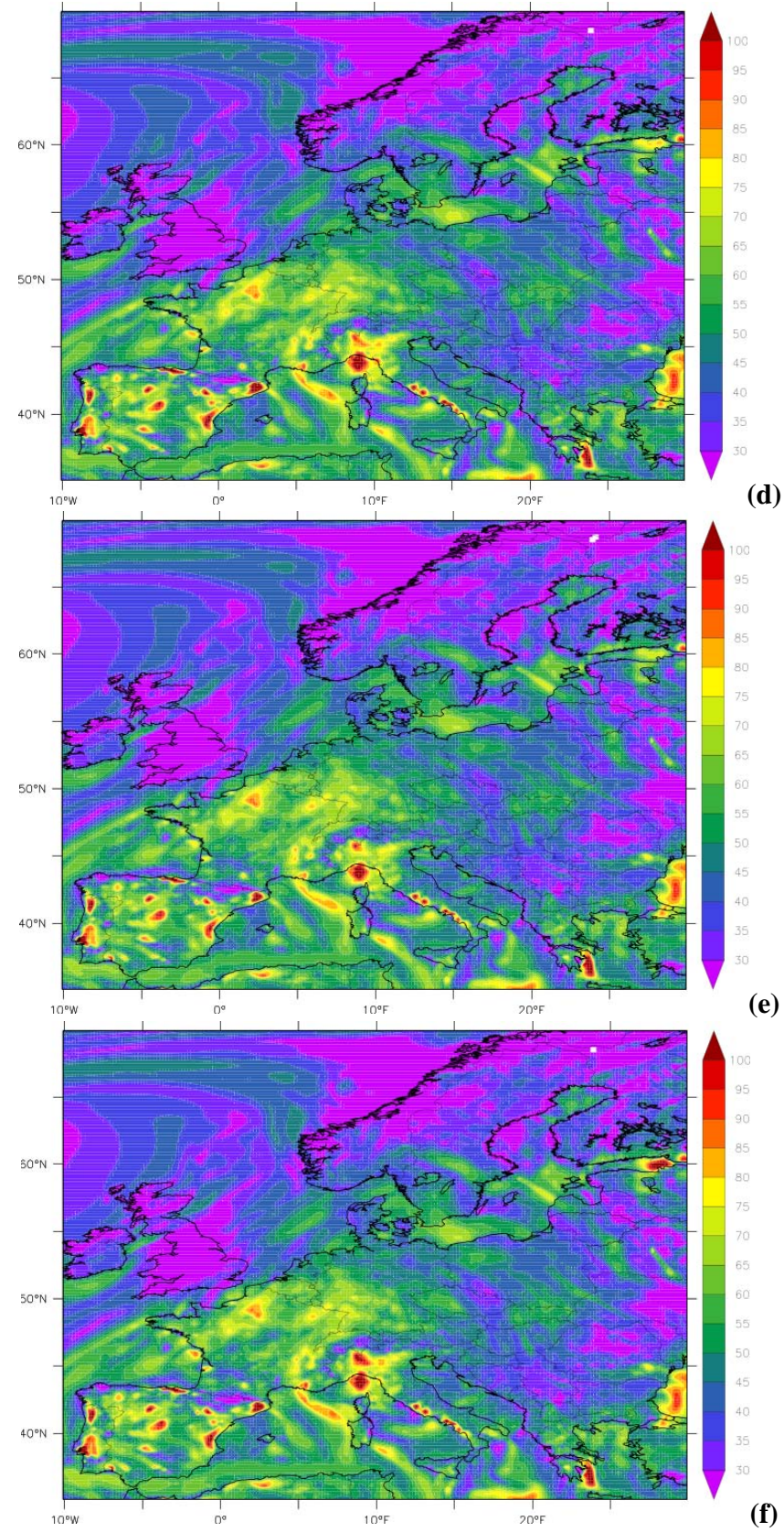

Fig. 9. Surface ozone concentration (in ppb) on the 7 July 2010 at 14:00:00 UTC for the (d) STD simulation, (e) LXY simulation, (f) STDLXY simulation (continued).

\subsection{BECM determination from a posteriori diagnostics}

Desroziers et al. (2007) introduced simple consistency diagnostics to check covariances of observation error, background error and estimation error in the observations space. These diagnostics are easily obtained since they use quantities available after the analysis, such as observed values and their background and analysis counterparts in the observations space. The consistency diagnostic on background errors can be formulated as

$\mathbf{H B H}^{T}=\mathbb{E}\left[\mathbf{d}_{b}^{a}\left(\mathbf{d}_{b}^{o}\right)^{T}\right]$,

with $\mathbf{d}_{b}^{a}$ the analysis-minus-background differences, $\mathbf{d}_{b}^{o}$ the innovation vector (i.e. the difference between observations and their background counterparts) and $\mathbf{H}$ the matrix corresponding to the linearized version of the observation operator. Note that this diagnostic does not produce information directly on the BECM but on its projection in the observations space.

Equation (12) must be satisfied if the BECM and OECM are optimum. So, generally, its left-hand side and its righthand side are calculated separately and compared. Each side of this equation is a matrix and a common method to compare them is to compare their traces. If the traces differ, a correction can be applied to the BECM to ensure identical traces and the analysis is repeated. The process can be iterated until a convergence is obtained.

Because of the expectation in the right-hand side of Eq. (12), the calculation must be done on an ensemble of data assimilation experiments in order to be significant and stable. But this turns out to be as computationally expensive as an ensemble approach.

A posteriori diagnostics are usually used to check the consistency of the BECM and the OECM. But since in our application the $\mathbf{H}$ observation operator is almost constant from one cycle to another, we replaced the computation of the expectation by an average over several assimilation cycles. We also assume that the standard deviation of the background error is of the same order as the diagonal of $\mathbf{H B H}^{\mathbf{T}}$ (which is the BECM projected in the observations space). Thus, the background standard deviation in the observations space can be written as

$$
\begin{aligned}
\operatorname{diag}\left(\Sigma_{\mathbf{H}}\right) & \simeq \operatorname{diag}\left(\mathbf{H B} \mathbf{H}^{\mathbf{T}}\right), \\
& \simeq \sqrt{\mathbb{E}\left[\mathbf{d}_{b}^{a}\left(\mathbf{d}_{b}^{o}\right)^{T}\right]}, \\
& \simeq \sqrt{\frac{1}{m} \sum_{j=1}^{m} \mathbf{d}_{b}^{a}\left(c_{j}\right)\left[\mathbf{d}_{b}^{o}\left(c_{j}\right)\right]^{T}}
\end{aligned}
$$

where $m$ is the number of cycles and $\Sigma_{\mathbf{H}}$ is made up of the $\Sigma$ values from observation grid-point locations.

Similarly, the observation error covariance matrix can be diagnosed and formulated as

$$
\begin{aligned}
\operatorname{diag}(\mathbf{R}) & =\sqrt{\mathbb{E}\left[\mathbf{d}_{a}^{o}\left(\mathbf{d}_{b}^{o}\right)^{T}\right]}, \\
& \simeq \sqrt{\frac{1}{m} \sum_{j=1}^{m} \mathbf{d}_{a}^{o}\left(c_{j}\right)\left[\mathbf{d}_{b}^{o}\left(c_{j}\right)\right]^{T}}
\end{aligned}
$$

where $\mathbf{d}_{a}^{o}$ is the observations-minus-analysis differences.

The correlations in the $\mathbf{H B H} \mathbf{H}^{\mathbf{T}}$ matrix are the background error correlations projected into the observations space. As usually the observation grid is too sparse compared to the 
model grid, it would be difficult to derive the background error correlation between two neighbouring model grid points from the background error correlation between two observation locations. Thus, using the $\mathbf{H B H}^{\mathbf{T}}$ matrix to estimate the background error correlation remains a difficult task and is out of the scope of the paper.

The above example of the use of a posteriori diagnostics to estimate the standard deviations of background and observation errors is an efficient alternative to an ensemble approach. This is especially true because its computational cost is negligible compared to the one of the ensemble method with the posteriori diagnostics computed over several assimilation cycles.

\section{Numerical experiments}

The numerical experiments described in this article have been done in the context of the European MACC project (MACC, 2010). Among the main MACC objectives is to provide information services covering European air quality. Involved in the MACC project, Cerfacs has adapted the Mocage-Valentina data assimilation system to produce daily analysis of air quality over Europe. Several chemical species are studied within MACC but we focused on surface ozone $\left(\mathrm{O}_{3}\right)$ in this study. Ozone is actively involved in atmospheric chemistry, and results from the transformation of chemical precursors by chemical and photochemical reactions. In the troposphere, ozone is one of the most relevant atmospheric pollutant indicator (Delmas et al., 2005; Finlayson-Pitts and Pitts, 2000).

\subsection{The chemistry transport model}

Our simulations of tropospheric and lower stratospheric chemical composition over Europe are performed with the Mocage multi-scale chemistry and transport model developed at Météo-France (Peuch, 1999). Mocage covers a large range of applications dealing with the study of interactions between chemistry and climate or the modelling of the tropospheric chemistry at a regional scale. Mocage uses meteorological fields (e.g. wind, humidity and temperature) to compute the transport of chemical elements, the chemical and photo-dissociation rates, or the exchanges between atmosphere and the surface. It describes the evolution of the modelled chemistry species and their horizontal and vertical transport in the troposphere and in the stratosphere (Josse, 2004). Among the different chemical schemes available within Mocage, we chose the RACMOBUS. RACMOBUS is a combination of the RACM chemical scheme dedicated to the troposphere (Stockwell et al., 1997) and the REPROBUS scheme dedicated to the stratosphere (Lefevre et al., 1994). It describes the evolution of 118 modelled chemistry species. The air quality version of Mocage uses several nested domains. The largest domain is global with a

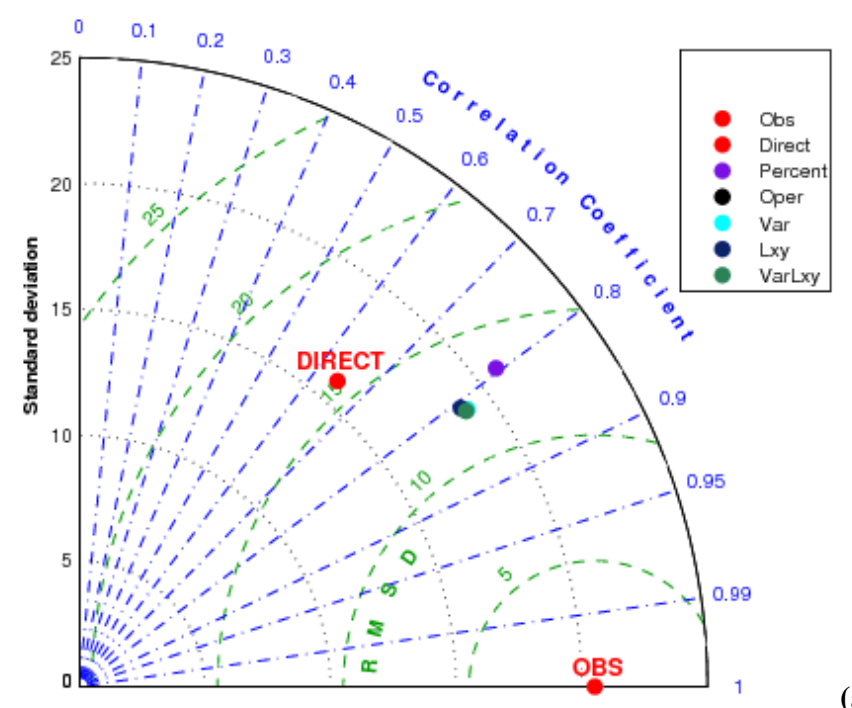

(a)

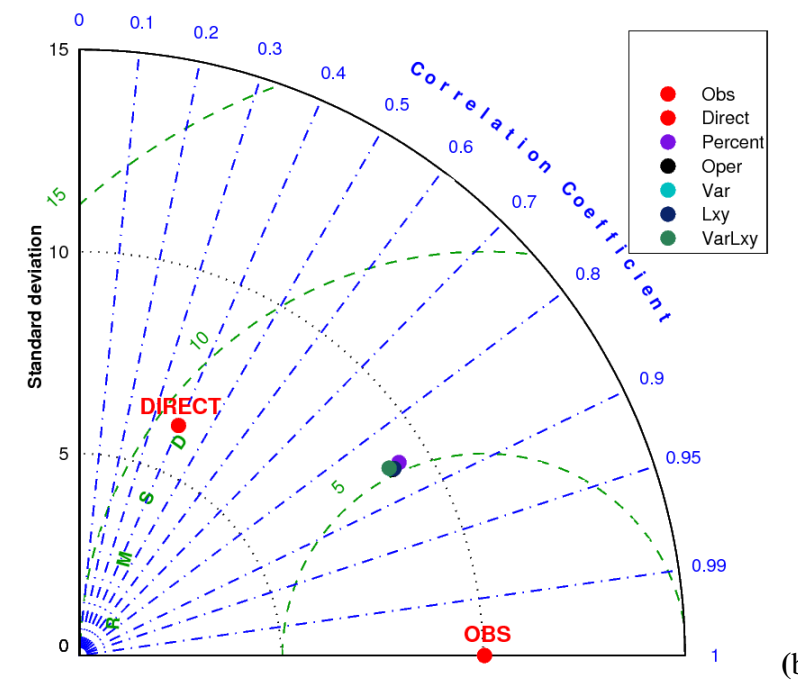

(b)

Fig. 10. Taylor diagram for several experiments studied, statistics averaged in the period between 1 and 10 July 2010 (a) and 1 and 10 December 2010 (b) for validation stations.

horizontal resolution of $2^{\circ} \times 2^{\circ}$. It provides the boundary conditions for the regional domain. In our study the regional domain covers a part of Western Europe from longitudes $16^{\circ} \mathrm{E}$ to $36^{\circ} \mathrm{W}$ and latitudes $32^{\circ} \mathrm{S}$ to $72^{\circ} \mathrm{N}$ with a horizontal resolution of $0.2^{\circ} \times 0.2^{\circ}$. For the two domains, the vertical resolution has 47 levels from the ground-level to approximately $35 \mathrm{~km}$.

\subsection{The data assimilation system}

Mocage is coupled to the Valentina assimilation system developed at Cerfacs (Buis et al., 2006). Mocage-Valentina is used for a number of applications to analyse the chemical composition of the atmosphere (Massart et al., 2012; Claeyman et al., 2010; Barret et al., 2008; Massart et al., 2009; 

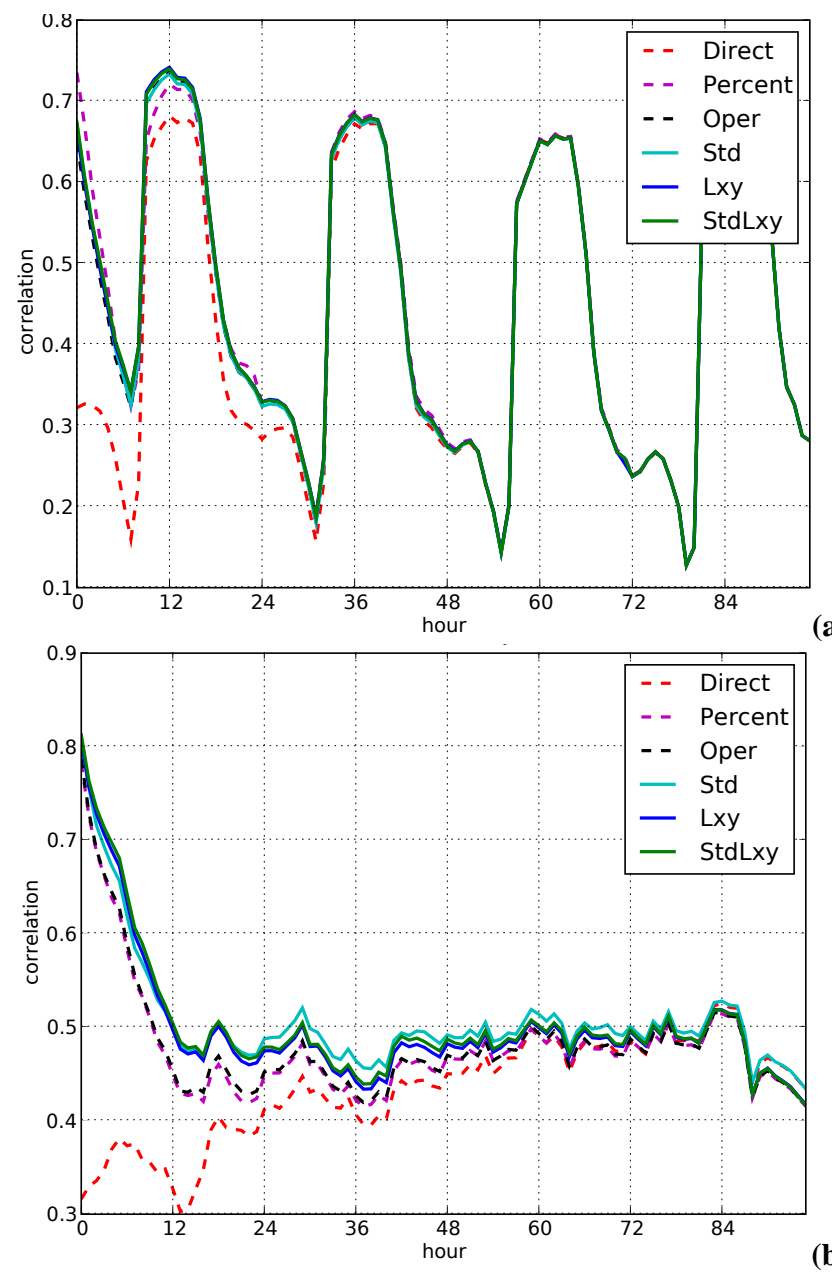

(a)

Fig. 11. Time series of the correlation between forecasts of experiments studied and assimilated observations, statistics on six fourdays forecasts in the period between 1 and 10 July 2010 (a) and 1 and 10 December 2010 (b).

El Amraoui et al., 2010). But even if some of the previous studies performed with Mocage-Valentina were dedicated to regional aspects of the atmospheric chemical composition, they were always done with Valentina coupled with Mocage in a configuration using only its global domain. To have a regional analysis at a better resolution than the one we could extract from an analysis performed at the global scale, a specific version of Valentina has been implemented. First Valentina was coupled with the regional domain of Mocage instead of its global domain. This allowed for the performance of analysis only in the regional domain of Mocage. Used surface measurements are performed each full hour. To avoid any problem we previously encounter with the 3D-Var FGAT method when the dynamics are rapid (Massart et al., 2010), we decided to use a simple 3D-Var method that produces an analysis each full hour when the data are available. Thus for each day, we have 24 successive analyses.

\subsection{Ground-based stations}

The ozone ground-based stations used in this study are the ones used within MACC (Airbase, 2011). They are located in Western Europe, mainly in France, Germany, Italy, England and Belgium (Fig. 1). We have chosen to study two periods, one in summer and the other in winter for the year 2010. The number of reporting stations depends on the period studied. More ozone data are reported during the summer period, especially in Italy, France, Sweden, Poland and Greece. The number of hourly observations varies in summer (resp. in winter) between 415 (resp. 382) and 739 (resp. 687) over Europe. If several stations give measurements in the same grid cell of the model mesh during the same hour, the median value of the ozone concentrations given by these stations is assimilated. This value is allocated to the average position of all the stations used. Not all the stations provide observations every hour.

\section{Estimate of the ensemble-based BECM for the surface ozone}

We have used the ensemble method combined with the previously described variational assimilation to estimate an ensemble-based BECM. In the following section, the characteristics of the performed experiments are detailed and the results are discussed.

\subsection{Characteristics of the case study}

Two opposite seasons are studied in order to account for seasonal variability, a summer period between the 1 and 10 July 2010 and a winter period between the 1 and 10 December 2010. For the two periods an ensemble of $10 \times 10 \times 24$ realizations is created representing 10 members during 10 days of simulation each day producing 24 analyses and forecasts. The 10 members are obtained by running in parallel the data assimilation system 10 times with perturbed emissions and perturbed observations for each member. The emissions used for each member are equal to the reference emissions multiplied by a global coefficient. The coefficient is constant for the whole day but differs from one day to another and from one member to another. It is randomly chosen in a Gaussian distribution with an average of one and a standard deviation of 50\%. In addition, the assimilated observations of each member are derived from the reference observation plus a centred Gaussian noise with a standard deviation consistent with the OECM. The OECM and BECM used to build the ensemble are based on simple formulations. As the two periods studied were already analysed, we used a posteriori diagnostics from Eqs. (13) and (14) to obtain these matrices. The OECM matrix was assumed to be diagonal with the same value for all the measurements, its standard deviation was found equal to $5.68 \mathrm{ppb}$ (parts per billion) in summer and to $4.79 \mathrm{ppb}$ in winter. For the BECM, a value for the standard 
deviation is obtained for each observation location from the a posteriori diagnostic and this value is assigned to the grid cell containing the observation. An average of the standard deviation is also computed and assigned to all the grid cells that do not contain any observation. Then a running average of five grid cells is applied to each grid point of the whole domain. The standard deviation obtained is on average larger during the summer period than during the winter period (Fig. 2), with a mean value of $4.65 \mathrm{ppb}$ in summer and $3.38 \mathrm{ppb}$ in winter. The correlations used in the BECM are difficult to obtain with a posteriori diagnostics. We thus used homogeneous and isotropic correlations by setting their length-scales to $45 \mathrm{~km}$ in the latitude and longitude directions. With this ozone value, variations are reflected over approximately two contiguous grid cells in each direction.

The 240 realizations obtained with the configuration described above allow the calculation of the length-scales and the standard deviation matrix using Eqs. (9) and (7), respectively.

\subsection{The ensemble-based correlation matrix estimate}

From the previously described ensemble, we first diagnose the $\mathbf{C}$ correlation matrix of the background errors. As we formulate it from length-scales, we have tested the two formulations to calculate the length-scales, i.e. the Gaussian lengthscales formula (Eq. 9) and the Belo Pereira and Berre formula (Eq. 10). As first results from the two formulations provided similar values for the length-scales, we chose to use only the Gaussian formula of Eq. (9).

Atmospheric flows have generally different characteristic scales in the two horizontal directions and in the vertical. Since the correlation structures of the background error are linked to the atmospheric flow, it is essential to study the correlations in all directions. Nevertheless, we only focused on the horizontal direction. Diagnosing the horizontal lengthscales requires the knowledge of the correlation $\rho(\delta)$. It is calculated in the two horizontal directions from Eq. (8). The east-west correlation is computed using the correlation between a point and its eastern neighbour. The north-south correlation is computed with the correlation between a point and its North neighbour.

The ensemble-diagnosed length-scales show that the horizontal correlations are non-isotropic during summer, mainly stretched in the north-south direction (Figs. 3, 4a). The average value of length-scales is $60 \mathrm{~km}$ in the north-south direction and $48 \mathrm{~km}$ in the east-west direction. During winter, the horizontal length-scales are more isotropic (Fig. 4b). The average value of length-scales is $56 \mathrm{~km}$ in the north-south direction and $52 \mathrm{~km}$ in the east-west direction. The values of the length-scales depend on many parameters such as wind, orography, type of grid cell (i.e. rural, urban, suburban). For example, results show that the largest length-scales are located in areas where a strong wind appears (i.e. in the Rhone valley or over Eastern Europe, figure not shown).
The isotropy is lost during summer. Larger north-south length-scales are in fact linked to the photochemical effect. At mid-latitude all the grid points along a given meridian have a zenith angle in the same range at a given time that implies similar photochemical effects for all these grid points, especially in summer when the photochemistry is a major component of the ozone evolution. These grid points are then correlated by the model and if we assume similar correlation in the model and its errors, then we can explain that forecast errors are more correlated along the meridional direction during summer. Consequently, the correlation of the forecast error is mainly isotropic, during the summer photochemical effects tending to produce anisotropy by stretching the correlation in the north-south direction.

The length-scale values are relatively high over Ireland, Eastern Europe, some oceanic regions and in the vicinity of domain boundaries (Fig. 3). This is related to the way the ensemble is built. First, in the vicinity of domain boundaries, when the wind is such that concentrations are influenced by boundary conditions, we lost some variability. This is for example the case for the region located West of the United Kingdom. In the upper left corner of the domain, the high values could be explained by a second phenomenon. In regions without observations (like oceans or Eastern Europe) the ensemble has a lower variability because of the lack of constraint by the perturbed observations. The only source of variability comes there from the perturbed emissions. And in the region of low emissions like the North West part of the North Sea, due to the low amount of emissions, perturbed emissions do not bring variability. This is less the case in some other oceanic regions where the ships produce $\mathrm{NO}_{\mathrm{x}}$ emissions that play a role in the ozone chemistry. In all the regions where the variability is low, the different simulations from all the members are thus very similar, which give a high correlation between them and results in large length-scales. To avoid too huge length-scale values, we have chosen to limit them to $200 \mathrm{~km}$. Note that we also have high values for the length-scales in the Western part of the Strait of Gibraltar. There the ensemble of simulations are probably similar due to the dynamics, the strong wind advecting ozone from the Mediterranean Sea (figure not shown).

From the members of the ensemble, hourly average statistics are calculated to obtain the diurnal variation of the ensemble-based BECM. Note that these statistics are now computed over 100 realizations. They show that the diurnal cycle of the ensemble-diagnosed length-scale is evident during a photochemically active period. For example in the grid cell of Paris, the length-scale values are high during summer when the ozone concentration is large (between 9:00:00 UTC to 16:00:00 UTC, Fig. 5a), between $32 \mathrm{~km}$ and $58 \mathrm{~km}$ (against $35 \mathrm{~km}$ and $43 \mathrm{~km}$ for winter). During winter ozone variations are mainly induced by transport, so the diurnal length-scales variations are small (Fig. 5a). During the summer period, the difference between north-south and west-east length-scales is clearly visible. This is also induced 
by the ozone photochemistry. In the Rhone valley during winter, the high north-south length-scales compared to the observed average values (Fig. 5b) may be due to the rapid ozone transport associated to the Mistral (a high North-South wind very common in the Rhone valley).

\subsection{The ensemble-based standard deviation matrix estimate}

We have also used the ensemble of backgrounds created from the different members of the ensemble approach to calculate the standard deviation part of the BECM with Eq. (11). The ensemble-diagnosed standard deviations are inhomogeneous over Europe (Fig. 6). As already explained in the previous section, where there is no constraint by the perturbed observations and near the boundaries of the domain (like Eastern Europe) or over a region with low emissions (like North Sea), we have low variability in the ensemble that results in low standard deviation. Note that over the Mediterranean Sea, in spite of the absence of observations, we produce some variability thanks to the perturbation of the emissions (from ships). Other geophysical features appear such as over the Alps, the Rhone valley and the largest cities in Spain and Italy during summer. The same structures appear over Europe during winter with a smaller average of the standard deviations, $4.85 \mathrm{ppb}$ (against $5.05 \mathrm{ppb}$ for summer). Larger standard deviations during the summer season could be due to high ozone concentration and variability. The anticyclonic condition over Europe during this period (figure not shown) leads to this chemical situation. A diurnal variation is observed during summer with an increase during the day. During winter, the variations of the standard deviations are smooth and the diurnal cycle is not visible (Fig. 7).

We can remark that the structure of the standard deviations obtained with the ensemble approach over Europe presents much higher spatial variations than the ones used to build the ensemble members (i.e. obtained with the a posteriori diagnostics shown in Fig. 2). The values estimated with our ensemble approach are larger than the diagnosed standard deviations values. We have likely chosen too large a standard deviation to perturb the emissions used to create the ensemble members. Thus, the standard deviations from the ensemble approach are too large.

\section{Impact of the BECM on surface ozone}

The ensemble-based diagnosed BECM indicates that this error is space- and time-dependent with sometimes large diurnal variations. So a time-dependent BECM should be adopted in our system to ensure a good surface ozone analysis. To measure the impact of such a time-dependent matrix and the impact of each of its components on the analysis of surface ozone over Europe, we performed the following study. Five experiments were performed based on BECMs modelled using for its components a simple formulation, a posteriori diagnostics, or ensemble-based estimations. Statistics on the five analyses and five forecasts from the five experiments are computed against a selection of validation stations. They are also used to estimate the most relevant configuration of the BECM for our case study.

\subsection{The BECM experiments}

Five experiments that differ only by the choice of the BECM configuration have been conducted. These experiments have been chosen to identify the influence of each BECM parameters (i.e. the length-scales and the standard deviations). These experiments are the following:

- PERCENT: the standard deviations of the BECM used for this experiment are taken as a percentage of the background, i.e. $20 \%$. This makes the BECM timedependent as the background changes. The horizontal length-scales are homogeneous in latitude and longitude. This value is constant and set to $45 \mathrm{~km}$ for each grid point and has no diurnal variation.

- OPER: the standard deviations used to build the BECM are derived from the a posteriori diagnostics computed in the month before the period studied (Desroziers diagnostics detailed in Sect. 2). The BECM is timeindependent. As in the PERCENT experiment, the horizontal length-scales are taken as homogeneous and equal to $45 \mathrm{~km}$. This experiment is the operational (OPER) configuration routinely used within the European MACC project.

- STD: the standard deviations used in the BECM for this experiment are those which were estimated from the ensemble method described in the previous section. As in the Oper and the Percent experiments, the horizontal length-scales are equal to $45 \mathrm{~km}$ in latitude and in longitude.

- LXY: in this experiment, the horizontal length-scales used to model the BECM are set to the values obtained from the ensemble-based estimation. The correlation matrix is time- and space-dependent. The standard deviations used in the BECM are the same as the OPER experiment.

- STDLXY: in this experiment, the BECM is modelled using both the length-scales and the standard deviations which were estimated from the ensemble of realizations described in the previous section. This experiment corresponds to a fully time-dependent BECM.

To evaluate the impact of these five experiments, we also used the Mocage run without assimilation. This simulation is our reference and is called the DIRECT experiment.

The characteristics of the different experiments are summarized in Table 1. 
Table 1. Summary of the experiments used to identify the influence of the BECM parameters. "diag." means that the parameter was calculated from a posteriori diagnostics; "ens." means that the parameter was calculated from an ensemble of realizations.

\begin{tabular}{ccccccc}
\hline & & \multicolumn{5}{c}{ Experiments } \\
& Direct & PERCENT & OPER & StD & LXY & StDLXY \\
\hline Length-scale & - & $45 \mathrm{~km}$ & $45 \mathrm{~km}$ & $45 \mathrm{~km}$ & ens. & ens. \\
\hline Std & - & $20 \%$ & diag. & ens. & diag. & ens. \\
\hline
\end{tabular}

Table 2. Coordinates of the validation stations; $1-5$ both for summer and winter periods, $6-10$ only for summer period, 11 only for winter period; FR for France and GE for Germany.

\begin{tabular}{ccccc}
\hline $\mathbf{1}$ & $\mathbf{2}$ & $\mathbf{3}$ & $\mathbf{4}$ & $\mathbf{5}$ \\
$\mathbf{F R}$ & $\mathbf{F R}$ & $\mathbf{F R}$ & $\mathbf{F R}$ & $\mathbf{G E}$ \\
$(47.42,-0.61)$ & $(49.16,6.65)$ & $(49.33,6.27)$ & $(49.35,0.11)$ & $(50.43,12.61)$ \\
\hline $\mathbf{6}$ & $\mathbf{7}$ & $\mathbf{8}$ & $\mathbf{9}$ & $\mathbf{1 0}$ \\
$\mathbf{F R}$ & $\mathbf{F R}$ & $\mathbf{F R}$ & $\mathbf{F R}$ & $\mathbf{G E}$ \\
$(43.45,4.93)$ & $(43.65,7.20)$ & $(47.27,-2.34)$ & $(47.74,7.30)$ & $(51.8,10.62)$ \\
\hline $\mathbf{1 1}$ & & & & \\
$\mathbf{F R}$ & & & & \\
$(43.51,4.98)$ & & & & \\
\hline
\end{tabular}

\subsection{Assimilated stations}

From the MACC ozone ground-based stations presented in Sect. 3, a selection of station was done. A classification by type of stations (i.e. urban, suburban and rural) has been developed at Centre National de Recherches Météorologiques (CNRM) by Joly and Peuch (2012). This classification in ten classes is based on the measurement data itself, using all the data available in the European AirBase data set and the French data set named BDQA (Base De données de Qualité de l'Air, i.e. Air Quality Data Base). Each class allows to group past time series of measured pollutants that are homogeneous from the point of view of their statistical properties. Thanks to this classification we retain exclusively rural stations in the assimilation process. Rural stations give a relevant representation of large-scale conditions because they are less influenced by local phenomena (e.g. emissions).

The ozone rural ground-based stations are mainly located in Western Europe. An example of rural station locations is shown in Fig. 8. The number of hourly rural observations varies in summer (resp. in winter) between 206 (resp. 152) and 255 (resp. 247) over Europe.

\subsection{Validation stations}

From the rural selection of assimilated stations, some stations were excluded from the assimilation process to serve as independent validation stations. We selected a validation station if its grid mesh contains more than three stations. The number of these validation stations depends on the season studied: 10 during summer and 6 during winter (red points in
Fig. 8). The coordinates of these stations are given in Table 2. Each day, the observations from the validation stations vary from 138 to 167 during summer, and from 72 to 95 during winter.

\subsection{Results for the assimilated stations}

The daily correlation between the analysis of each experiment and the assimilated observations are given in Tables 3 and 4 for the two periods studied. The correlation of the five assimilation experiments is relatively high (around 0.9) and is always larger than the correlation obtained with the direct Mocage run (i.e. the DIRECT experiment). The values of the correlation from the DIRECT experiment show large seasonal variations. During the winter period, the daily correlation is sometimes very low, as an example around 0.2 for the period 1 to 10 December 2010. This is likely due to the difficulty of the Mocage model to account for low ozone concentrations. However, during the summer period, high peaks of ozone concentration are well modelled and the correlation is higher (around 0.7).

The five assimilated experiments have different behaviours: an experiment that gives the largest correlation for one day, does not necessarily give the largest the day after. But, in general, during the summer period the largest correlation is found in the PERCENT and STDLXY experiments, whereas during the winter period the STD experiment has the largest correlation. The PERCENT experiment gives less confidence to the background state than the other experiments with a smaller standard deviation of the background error. That is likely why the analysis from the PERCENT experiment is closer to the assimilated observations. 
Table 3. Daily correlation between analysis of the experiments studied and assimilated observations, statistics during the period between 1 and 10 July 2010; the largest daily correlation is in bold.

\begin{tabular}{lcccccc}
\hline & \multicolumn{5}{c}{ Summer correlation } \\
& DIRECT & PERCENT & OPER & STD & LXY & STDLXY \\
\hline 20100701 & 0.7121 & $\mathbf{0 . 9 1 2 0}$ & 0.8915 & 0.8989 & 0.8951 & 0.9056 \\
20100702 & 0.7242 & $\mathbf{0 . 9 0 0 3}$ & 0.8929 & 0.8939 & 0.8936 & 0.8966 \\
20100703 & 0.6183 & 0.8923 & 0.8898 & 0.8940 & 0.8924 & $\mathbf{0 . 8 9 4 5}$ \\
20100704 & 0.5939 & 0.8924 & 0.8779 & 0.8864 & 0.8900 & $\mathbf{0 . 9 0 5 1}$ \\
20100705 & 0.6376 & $\mathbf{0 . 9 3 8 2}$ & 0.9205 & 0.9236 & 0.9254 & 0.9248 \\
20100706 & 0.5791 & $\mathbf{0 . 9 3 0 6}$ & 0.9075 & 0.9115 & 0.9111 & 0.9168 \\
20100707 & 0.6905 & $\mathbf{0 . 9 4 9 3}$ & 0.9311 & 0.9366 & 0.9372 & 0.9387 \\
20100708 & 0.6641 & 0.8924 & 0.8860 & 0.8879 & 0.8902 & $\mathbf{0 . 8 9 4 5}$ \\
20100709 & 0.6972 & 0.9044 & 0.9056 & $\mathbf{0 . 9 1 1 2}$ & 0.9056 & 0.9099 \\
20100710 & 0.7184 & $\mathbf{0 . 9 2 6 9}$ & 0.9016 & 0.9059 & 0.9030 & 0.9089 \\
\hline
\end{tabular}

Table 4. Same as Table 3 with statistics on the period between 1 and 10 December 2010.

\begin{tabular}{lcccccc}
\hline & \multicolumn{5}{c}{ Winter correlation } \\
& DIRECT & PERCENT & OPER & STD & LXY & STDLXY \\
\hline 20101201 & 0.1950 & 0.8646 & 0.8734 & 0.9085 & 0.8981 & $\mathbf{0 . 9 0 9 4}$ \\
20101202 & 0.2864 & 0.8569 & 0.8834 & 0.9200 & 0.9138 & $\mathbf{0 . 9 2 7 5}$ \\
20101203 & 0.2705 & 0.8901 & 0.8977 & $\mathbf{0 . 9 2 1 6}$ & 0.8977 & 0.9100 \\
20101204 & 0.3481 & 0.8847 & 0.8909 & $\mathbf{0 . 9 1 2 9}$ & 0.9037 & 0.9095 \\
20101205 & 0.4140 & $\mathbf{0 . 8 9 3 8}$ & 0.8683 & 0.8833 & 0.8667 & 0.8756 \\
20101206 & 0.5258 & 0.8795 & 0.8677 & $\mathbf{0 . 8 8 1 6}$ & 0.8619 & 0.8673 \\
20101207 & 0.6549 & 0.8918 & 0.8986 & $\mathbf{0 . 9 1 1 0}$ & 0.8841 & 0.8965 \\
20101208 & 0.6361 & $\mathbf{0 . 9 1 1 2}$ & 0.8904 & 0.9027 & 0.8825 & 0.8946 \\
20101209 & 0.4118 & 0.8783 & 0.8717 & $\mathbf{0 . 8 9 0 5}$ & 0.8710 & 0.8822 \\
20101210 & 0.2270 & 0.8617 & 0.8485 & $\mathbf{0 . 8 8 7 5}$ & 0.8535 & 0.8793 \\
\hline
\end{tabular}

The two LXY and STDLXY experiments also produce high correlation values compared to assimilated data. As the length-scales of the BECM are larger for these experiments than the length-scales of the others, we deduced that increasing the correlation length-scales of the background error allows to better represent the assimilated data.

Note that we only discuss in this paper the correlation between different sets of observations. We do not discuss other statistical diagnostics. But we nevertheless systematically checked the root-mean-square statistic (RMS), as we found a similar behaviour for the correlation and the RMS, the results on the RMS will not be discussed in this paper.

Even if statistical results compared to assimilated data are close for each experiment, we observed significant differences on individual analysis. For example, surface ozone fields over Europe on the 7 July 2010 at 14:00:00 UTC are represented in Fig. 9 for the six experiments studied. Common features are observed: the highest ozone concentrations arise where the assimilated stations are located and high peaks of ozone are visible over the most polluted places (e.g. over big cities). The DIRECT experiment mostly underestimates the ozone concentrations on these polluted places. Comparing the DIRECT experiment with the various analyses shows that the Mocage model tends to produce more ozone at the surface than the analyses, even over places where the analyses are not constrained by the observations (e.g. in England and Eastern Europe, figures not shown). In the experiments with a horizontal length-scales equal to $45 \mathrm{~km}$ (i.e. the Percent, OPER and STD experiments), large differences are observed in the ozone fields in the vicinity of large cities, such as over Paris, Milano, Madrid or Valencia. The ozone fields agree better when the standard deviations matrix is diagnosed with a posteriori diagnostics (i.e. the OPER experiment) or is estimated from the ensemble of realizations (i.e. the STD experiment). The distribution of ozone over the Alps and urban cities is also quite similar when the ensemble-based correlation matrix is used (i.e. the LXY and STDLXY experiments). The surface ozone in the polluted areas varies between the five experiments and depends on both correlation and standard deviation matrices.

\subsection{Results for the validation stations}

A Taylor Diagram is used to compare the analyses from the six experiments (i.e. the DIRECT and the five assimilated experiments) averaged over the 10-days period to the 
independent validation stations. This type of diagnostic summarises well the statistics of the analysed fields from the six experiments in comparison with the reference field obtained from the validation stations. The position of each point quantifies how closely the analysis pattern matches observations. The correlation of an analysis and the observation patterns is indicated on the radius of the diagram. The standard deviation of an analysis is proportional to the radial distance from the origin. The centred root-mean-square difference between an analysis and the observation pattern is proportional to the distance to the point on the $\mathrm{x}$-axis identified as "OBS".

All the experiments issued from the assimilation process have a better RMS and a standard deviation closer with respect to the observations than the DIRECT experiment. The 10-days correlations between analyses and the validation stations are lower than the correlations between analyses and the assimilated stations. This means that the analysis is not able to constrain the values in the grid-cells which are too far from the assimilated stations. The five experiments issued from assimilation have a large correlation, ranging from 0.8 to 0.85 (Fig. 10) during the two periods studied. The statistics of the five experiments are very similar during the winter period (Fig. 10b), but show more spread in summer (Fig. 10a). The largest correlation is obtained when the length-scales vary in the BECM. During summer the largest correlation is given by the VARLXY experiment and during winter by the LXY eXperiment. A systematic bias (i.e. the difference between the mean of the analysis and the mean of the observations) appears in the Mocage model, larger during the winter period than during the summer period $(-3.37 \mathrm{ppb}$ in winter against -1.62 in summer). The bias is reduced thanks to the five experiments issued from the assimilation process during the winter period but the bias is larger with these experiments during the summer period (figures not shown).

Those diagnostics clearly show that our assimilation process is efficient to bring the model closer to the observations than the direct simulation, but we cannot conclude at this stage which BECM configuration is the best.

\subsection{The impact of the BECM experiments on forecasts}

To assess the differences between the models used for the BECM, we also studied the impact of our experiments on the ozone forecasts. To this end, six forecasts of four days (i.e. using Mocage without an assimilation technique) are created for each assimilated experiment. The first forecast begins on the first day of the period studied; the second forecast begins the day 2 of the period studied; etc. For each forecast, the initial conditions come from the analysis. Thus, six forecasts of $96 \mathrm{~h}$ are obtained for each assimilated experiment.

In parallel, six four-day forecasts of the DIRECT experiment are obtained from the Mocage simulation without assimilation. The first forecast is made up of the days 1 to 4 of the Mocage simulation; the second forecast is made up of the days 2 to 5 of the Mocage simulation; etc.
The hourly correlations between the six $96 \mathrm{~h}$ forecasts and the assimilated observations are calculated. Thus, the value of the correlation at $1 \mathrm{~h}$ represents the mean of the correlations of the first hour of the six forecasts.

Improvements in ozone forecasts subsequent to the assimilation procedure were found as in the studies of Elbern and Schmidt (2001) and Blond and Vautard (2004). In our study, results indicate that the impact of the assimilation process persists longer during the winter period than the summer period: one day in summer and about three days in winter (Fig. 11). After that, forecasts from the five experiments from the assimilations are very similar to the DIRECT simulation. These results are consistent with the study of Blond and Vautard (2004) which shows that ozone analysis initialization improves the simulation for $24-48 \mathrm{~h}$ later and beyond that time the analysis becomes identical to the simulation without assimilation. During the first $12 \mathrm{~h}$, the influence of our assimilation is rapidly reduced for the winter period. In summer, large daily variations of the correlation coefficient are observed, but the analyses from the five experiments are all better than the direct simulation for the first day. During summer nights, Mocage does not model well the ozone evolution and gives low correlations (less than 0.2). During the winter period studied, the Mocage simulation starts with a correlation equal to 0.3 that grows the days after. As the correlation with the observations of the 1 December 2010 is very low (see Table 4), the mean of the six analyses is significantly reduced as the beginning of the $96 \mathrm{~h}$ forecast correlation, and the correlation profile of the Mocage simulation is not close to the profile of the other experiments.

\section{Conclusions and future work}

In this paper we have studied the impact on surface ozone analysis of a time-dependent background error covariances matrix. In our approach the BECM is constructed from a combination of a correlation and a standard deviations matrices, the correlations being characterized by length-scales. To evaluate our BECM model, we diagnose the standarddeviations and the length-scales using an ensemble approach and a posteriori diagnostics.

The diagnostics from the ensemble approach have shown that over Europe the length-scales and the standard deviations of the forecast error vary significantly spatially and temporally (both hourly and seasonally). These variations are larger during the summer period than during the winter period because the ozone concentration and its variations are photochemically driven. The values of the standard deviations estimated with the ensemble approach are larger than the diagnosed standard deviations with a posteriori diagnostics. The standard deviation used to perturb the emissions to create the ensemble members is likely too large.

We have performed several experiments that differ by the choice of the BECM configuration, in which the correlations 
and standard deviations matrices come from the ensemblebased diagnostics or from a posteriori diagnostics. The results from these experiments are compared to a simulation without assimilation, and to a simpler experiment in which the standard deviation is equal to a percentage of the background field and the length-scales are fixed. All the analyses from these experiments are very correlated to the observations, the data assimilated or the independent validation stations. Comparisons of the analyses with the validation stations show that the use of a BECM with time-dependent length-scales gives the largest correlation. All the analyses have a better correlation with the observations than the direct Mocage simulation, but the differences between them are small and we cannot conclude which BECM formulation is the most appropriate to the ozone simulation over Europe. The correlation depends on the period studied and may change from one day to another. Finally, the impact of the BECM formulation on four-days forecasts has been studied. Our assimilation process has a different time influence on the ozone forecasts depending on the period: one day during the summer season, three days during the winter season. For longer forecasts, the simulations from the analyses appear to have no added value compared to the direct Mocage simulation.

However our results have been obtained for rather limited periods. It remains to be seen how the present statistics would evolve for multi-year analyses. The impact of the BECM formulation has been also difficult to evaluate because the Mocage model shows a systematic bias in situations with low ozone concentration. So the impact of the analysis process is mostly to remove this bias, but this appears to be insufficient to improve the forecast beyond one day. It would be therefore interesting to evaluate the influence of the BECM formulation following the same methodology, but with other chemistry transport models with different error characteristics.

Acknowledgements. The authors would like to thank William Lahoz for the constructive comments on this paper.

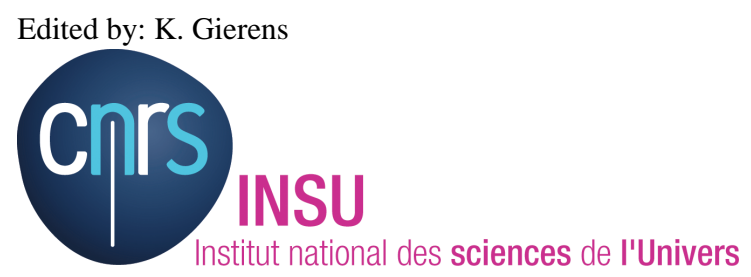

The publication of this article is financed by CNRS-INSU.

\section{References}

Airbase: The European air quality database (version 5), http://www.eea.europa.eu/data-and-maps/data/ airbase-the-european-air-quality-database-3, last access: 22 February 2012, 2011.

Barret, B., Ricaud, P., Mari, C., Attié, J.-L., Bousserez, N., Josse, B., Le Flochmoën, E., Livesey, N. J., Massart, S., Peuch, V.H., Piacentini, A., Sauvage, B., Thouret, V., and Cammas, J.P.: Transport pathways of $\mathrm{CO}$ in the African upper troposphere during the monsoon season: a study based upon the assimilation of spaceborne observations, Atmos. Chem. Phys., 8, 3231-3246, doi:10.5194/acp-8-3231-2008, 2008.

Belo Pereira, M. and Berre, L.: The use of an Ensemble approach to study the Background Error Covariances in a Global NWP model, Mon. Wea. Rev., 134, 2466-2489, 2006.

Blond, N. and Vautard, R.: Three-dimensional ozone analyses and their use for short-term ozone forecasts, J. Geophys. Res, 109, D17303, doi:10.1029/2004JD004515, 2004.

Blond, N., Bel, L., and Vautard, R.: Three-dimensional ozone data analysis with an air quality model over the Paris area, J. Geophys. Res, 108, 1993-1996, 2003.

Buehner, M.: Ensemble-derived stationary and flow-dependent background-error covariances: Evaluation in a quasi-operational NWP setting, Q. J. Roy. Meteorol. Soc., 131, 1013-1043, 2005.

Buis, S., Piacentini, A., and Déclat, D.: PALM: a computational framework for assembling high-performance computing applications, Concurrency and computation: practice and experience, 18, 231-245, 2006.

Claeyman, M., Attié, J.-L., El Amraoui, L., Cariolle, D., Peuch, V.H., Teyssèdre, H., Josse, B., Ricaud, P., Massart, S., Piacentini, A., Cammas, J.-P., Livesey, N. J., Pumphrey, H. C., and Edwards, D. P.: A linear CO chemistry parameterization in a chemistrytransport model: evaluation and application to data assimilation, Atmos. Chem. Phys., 10, 6097-6115, doi:10.5194/acp-10-60972010, 2010.

Coman, A., Foret, G., Beekmann, M., Eremenko, M., Dufour, G., Gaubert, B., Ung, A., Schmechtig, C., Flaud, J.-M., and Bergametti, G.: Assimilation of IASI partial tropospheric columns with an Ensemble Kalman Filter over Europe, Atmos. Chem. Phys., 12, 2513-2532, doi:10.5194/acp-12-2513-2012, 2012.

Constantinescu, E., Sandu, A., Chai, T., and Carmichael, G.: Ensemble-based chemical data assimilation. I: General approach, Q. J. Roy. Meteorol. Soc, 133, 1229-1243, 2007.

Daley, R.: Atmospheric data analysis, Cambridge atmospheric and space sciences series, Cambridge University Press, 457, 1991.

Delmas, R., Mégie, G., and Peuch, V.: Physique et chimie de l'atmosphère, Belin, 608, 2005.

Desroziers, G., Berre, L., Chapnik, B., and Poli, P.: Diagnosis of observation, background and analysis-error statistics in observation space, Q. J. Roy. Meteorol. Soc., 131, 3385-3396, 2007.

Desroziers, G., Berre, L., Pannekoucke, O., Stefanescu, S., Brousseau, P., Auger, L., Chapnik, B., and Raynaud, L.: Flowdependent error covariances from variational assimilation ensembles on global and regional domains, Hirlam Technical ReportY, 68, 2-22, 2008.

El Amraoui, L., Attié, J.-L., Semane, N., Claeyman, M., Peuch, V.H., Warner, J., Ricaud, P., Cammas, J.-P., Piacentini, A., Josse, B., Cariolle, D., Massart, S., and Bencherif, H.: Midlatitude stratosphere - troposphere exchange as diagnosed by MLS $\mathrm{O}_{3}$ 
and MOPITT CO assimilated fields, Atmos. Chem. Phys., 10, 2175-2194, doi:10.5194/acp-10-2175-2010, 2010.

Elbern, H. and Schmidt, H.: Ozone episode analysis by fourdimensional variational chemistry data assimilation, J. Geophys. Res., 106, 3569-3590, 2001.

Elbern, H., Schwinger, J., and Botchorishvili, R.: Chemical state estimation for the middle atmosphere by four-dimensional variational data assimilation: System configuration, J. Geophys. Res, 115, D06302, doi:10.1029/2009JD011953, 2010.

Finlayson-Pitts, B. and Pitts, J.: Chemistry of the upper and lower atmosphere: Theory, experiments, and applications, Academic Pr, 2000.

Joly, M. and Peuch, V.-H.: Objective classification of air quality monitoring sites over Europe, Atmos. Environ., 47, 111-123, 2012.

Josse, B.: Représentation des processus de transport et de lessivage pour la modélisation de la composition chimique de l'atmosphère à l'échelle planétaire, Ph.D. thesis, Université Paul Sabatier, Toulouse (UPS), 2004.

Lefevre, F., Brasseur, G., Folkins, I., Smith, A., and Simon, P.: Chemistry of the 1991-1992 stratospheric winter: Threedimensional model simulations, J. Geophys. Res., 99, 81838195, 1994.

MACC: Monitoring Atmospheric Composition and Climate, http: //www.gmes-atmosphere.eu/, (last access: 2 August 2012), 2010.

Massart, S., Cariolle, D., and Peuch, V.-H.: Vers une meilleure représentation de la distribution et de la variabilité de l'ozone atmosphérique par l'assimilation des données satellitaires, CRAS, 337, 1305-1310, 2005.

Massart, S., Clerbaux, C., Cariolle, D., Piacentini, A., Turquety, S., and Hadji-Lazaro, J.: First steps towards the assimilation of IASI ozone data into the MOCAGE-PALM system, Atmos. Chem. Phys., 9, 5073-5091, doi:10.5194/acp-9-5073-2009, 2009.
Massart, S., Pajot, B., Piacentini, A., and Pannekoucke, O.: On the merits of using a 3D-FGAT assimilation scheme with an outer loop for atmospheric situations governed by transport, Mon. Wea. Rev., 4509-4522, doi:10.1175/2010MWR3237.12010.

Massart, S., Piacentini, A., and Pannekoucke, O.: Importance of using ensemble estimated background error covariances for the quality of atmospheric ozone analyses, Q. J. Roy. Meteor. Soc., 138, 889-905, doi:10.1002/qj.971, 2012.

Pannekoucke, O. and Massart, S.: Estimation of the local diffusion tensor and normalization for heterogeneous correlation modelling using a diffusion equation, Q. J. Roy. Meteor. Soc., 134, 1425-1438, 2008.

Pannekoucke, O., Berre, L., and Desroziers, G.: Background-error correlation length-scale estimates and their sampling statistics, Q. J. Roy. Meteorol. Soc., 134, 497-508, 2008.

Peuch, V.-H., Amodei, M., Barthet, T., Cathala, M.-L., Josse, B., Michou, M., and Simon, P.: MOCAGE : Modèle de ChimieTransport à Grande Echelle, in: Acte de l'Atelier de Modélisation de l'Atmosphère, 33-36, 1999.

Reichle, R.: Data assimilation methods in the Earth sciences, Adv. Water Resour., 31, 1411-1418, 2008.

Stockwell, W., Kirchner, F., Kuhn, M., and Seefeld, S.: A new mechanism for regional atmospheric chemistry modeling, J. Geophys. Res., 102, 25847-25879, 10.1029/97JD00849, 1997.

Swinbank, R., Shutiev, V., and Lahoz, W.: Data assimilation for the earth system, 26, Springer Netherlands, 2003.

Talagrand, O.: Assimilation of observations, an introduction, J. Meteorol. Soc. Jpn, 75, 81-99, 1997.

Weaver, A. and Ricci, S.: Constructing a background-error correlation model using generalized diffusion operators, in: Proceedings of the ECMWF Seminar Series on Recent developments in atmospheric and ocean data assimilation, ECMWF, Reading, UK, 8-12, 2003. 